\title{
BMJ Open Do shorter delays to care and mental health system renewal translate into better occupational outcome after mental disorder diagnosis in a cohort of Canadian military personnel who returned from an Afghanistan deployment?
}

David Boulos, ${ }^{1}$ Mark A Zamorski ${ }^{1,2}$

To cite: Boulos D,

Zamorski MA. Do shorter delays to care and mental health system renewal translate into better occupational outcome after mental disorder diagnosis in a cohort of Canadian military personnel who returned from an Afghanistan deployment?. BMJ Open 2015;5:e008591. doi:10.1136/bmjopen-2015008591

- Prepublication history for this paper is available online. To view these files please visit the journal online (http://dx.doi.org/10.1136/ bmjopen-2015-008591).

Received 23 April 2015 Revised 30 October 2015 Accepted 2 November 2015

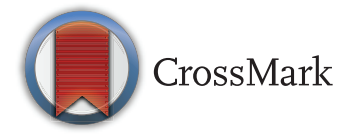

For numbered affiliations see end of article.

Correspondence to

David Boulos;

david.boulos@forces.gc.ca

\section{ABSTRACT}

Objective: Mental disorders in military personnel result in high rates of attrition. Military organisations have strengthened their mental health systems and attempted to overcome barriers to care in order to see better outcomes. This study investigated the roles of mental health services renewal and delay to care in Canadian Armed Forces (CAF) personnel diagnosed with mental disorders.

Design: Administrative data were used to identify a retrospective cohort of 30513 CAF personnel who deployed in support of the mission in Afghanistan. Study participants included 508 individuals with a mental disorder diagnosis identified from CAF medical records of a weighted, stratified random sample of 2014 individuals selected from the study cohort. Weighted Cox proportional hazards regression assessed the association of diagnosis era and delay to care with the outcome, after controlling for a broad range of potential confounders (eg, disorder severity, comorbidity). Taylor series linearisation methods and sample design weights were applied in generating descriptive and regression analysis statistics.

Primary outcome: The outcome was release from military service for medical reasons, assessed using administrative data for the 508 individuals with a mental disorder diagnosis.

Results: $17.5 \%$ (95\% Cl $16.0 \%$ to $19.0 \%$ ) of the cohort had a mental disorder diagnosis after an Afghanistan-related deployment, of which 21.3\% (95\% $\mathrm{Cl} 17.2 \%$ to $25.5 \%$ ) had a medical release over a median follow-up of 3.5 years. Medical release risk was elevated for individuals diagnosed before 30 April 2008 relative to those with recent diagnoses (adjusted HR $(\mathrm{aHR})=1.77(95 \% \mathrm{Cl} 1.01$ to 3.11$))$ and for individuals with a long delay to care (>21 months after return) relative to those with intermediate delays (8-21 months, aHR $2.47=(95 \% \mathrm{Cl} 1.28$ to 4.76$))$. Conclusions: Mental health services renewal in the CAF was associated with a better occupational outcome

\section{Strengths and limitations of this study}

- This study demonstrates that two modifiable factors, mental health system change and delay to care, have associations with medically related attrition rates among personnel with a mental disorder in a previously deployed Canadian military cohort. After controlling for potential confounding variables, the accumulation of change in the Canadian military's mental health system was determined to be associated with lower rates of medically related attrition. Long delays to care were associated with higher rates of medically related attrition.

- Disorder severity, diagnosis mix (specifically, post-traumatic stress disorder comorbid with a depressive disorder), being a non-commissioned service member, and being in certain occupational categories were also determined to be independently associated with medically related attrition rates.

- Diagnosis era was a proxy for changes in the Canadian Armed Forces (CAF) mental health system. It was not possible to identify precise implementation time points for the many concurrent mental health system changes, precluding identification of the drivers of the more favourable outcomes seen over time.

for those diagnosed with mental disorders. Longer delays to care were associated with a less favourable outcome.

\section{INTRODUCTION}

Since 2001, millions of military personnel have deployed to the conflicts in Southwest Asia; many have returned with mental health 
problems. ${ }^{1} 2$ In Canada, $13.5 \%$ of personnel who deployed in support of the mission in Afghanistan were diagnosed with a service-related mental disorder within 4 years after their return. ${ }^{2}$ For those in high-threat locations, rates approached $30 \%$ after 7 years. ${ }^{2}$

Mental health problems have a pronounced impact on military occupational fitness, ${ }^{3-17}$ which in turn can lead to attrition when personnel fail to meet stringent military occupational fitness requirements. Attrition is the largest single mental health-related cost for employers, ${ }^{18}$ and it has particular salience for military organisations, given their extensive and specialised training requirements.

Many factors influence adverse military occupational outcomes such as attrition. ${ }^{5-8} 1017{ }^{19-24}$ In addition to mental health problems, ${ }^{3-17}$ factors include age,${ }^{5-8} 10$ 19-21 24 gender, ${ }^{5-7} \quad 10 \quad 17$ 19-22 race/ethnicity, ${ }^{6} \quad 8 \quad 19$ rank, ${ }^{5} 101719202324$ component (Regular or Reserve Force),${ }^{19}$ years of service, ${ }^{10} 172324$ marital status, ${ }^{1022} 24$ element (Army, Navy or Air Force) ${ }^{521}$ and education. ${ }^{10}$

Research on factors influencing outcome among those with mental disorders is sparse. Associations have been seen with diagnosis, ${ }^{17} 23$ illness severity, ${ }^{23}{ }^{24}$ years of service, ${ }^{17} 2324$ combat occupation, ${ }^{17}{ }^{24}$ rank, ${ }^{17} 2324$ gender, ${ }^{17}{ }^{24}$ age, ${ }^{24}$ marital status, ${ }^{24}$ presence of comorbid conditions ${ }^{17}$ and treatment, ${ }^{17}$ factors that are mostly non-modifiable.

The Canadian Armed Forces (CAF) has reinforced its mental health system over the past 15 years. ${ }^{25}$ The number of mental health providers has more than doubled. Seven multidisciplinary centres now provide standardised assessment and treatment of service-related mental health problems. Lower levels of stigma and other barriers to care have been seen in comparison to other military organisations, ${ }^{26}$ perhaps as a result of de-stigmatisation efforts. $^{27}$ Extensive resilience and mental health training is embedded throughout individuals' careers and across the deployment cycle, which includes in-depth postdeployment mental health screening. ${ }^{28}$ The past decade has also seen policy changes that make it easier for personnel who recover from mental disorders to remain in uniform. Other nations have engaged in similar efforts. ${ }^{29} 30$

Mental disorders have been shown to be associated with attrition, ${ }^{13}{ }^{31}$ both before and after the current conflicts in Southwest Asia, but there are no formal assessments of changes in prognosis over time. Additionally, the temporal changes in the CAF mental health system that targeted reductions in barriers to care-seeking as one of its objectives were expected to impact those who would otherwise not seek care as well as reduce careseeking delays in others. However, delay to care can potentially act independently on medically related attrition, as it has been asserted that early care for post-traumatic stress disorder (PTSD) and other mental disorders has the potential to improve long-term prognosis. Unfortunately, there is very little research supporting this assertion. ${ }^{32}$
This study investigates risk factors for medically related attrition among personnel who deployed in support of the CAF mission in Afghanistan and were diagnosed with a mental disorder. We hypothesised that those who were diagnosed later in the course of the conflict and those who presented for care earlier would independently show more favourable outcomes.

\section{METHODS}

\section{Study population and sampling}

The study cohort consists of all CAF personnel $(\mathrm{N}=30$ 513) who initiated a deployment outside of North America and Europe in support of the Afghanistan mission from 1 October 2001 through 31 December 2008. Over the duration of the mission, 158 CAF personnel died on-duty. A weighted, stratified random sample of 2045 individuals was identified and medical records of 2014 were reviewed; medical records were inaccessible for 31 individuals before the end of the chart review phase of the study, either because they were in transition to a new location or were in active use for reasons other than treatment. The sampling strata were defined by deployment location and apparent mental health services use, strata relevant to the parent study's primary objective. $^{2}$

The current study was limited to 508 of the 2014 individuals whose chart review indicated that they had a mental disorder diagnosis dated after the start date of their first Afghanistan-related deployment.

\section{Data collection}

As discussed elsewhere ${ }^{2}$ deployment details came from administrative databases. Mental disorder diagnoses, mental disorder history and clinician-identified attributions to service were abstracted from medical records over the period of 22 June 2010 to 30 May 2011. The evaluations of service-related mental disorders are done in one of seven CAF regional mental health centres and they follow a common, collaborative assessment model that includes psychological testing and consultation with, at minimum, a psychiatrist and a psychologist. The clinicians comment on the association between the diagnosis and military service because this can have implications in terms of veterans' benefits.

Data on release from service, demographics and military characteristics came from administrative databases (extract date 15 December 2012). Data linkages were based on service number, a unique CAF-specific personal identifier.

\section{Outcome definition}

The primary outcome was release from military service for medical reasons (ie, medically related attrition). The broad categories for release, and their representation in the cohort, include misconduct $(0 \%)$, unsatisfactory service $(0 \%)$, service completed $(3.7 \%)$, medical $(5.4 \%)$ and voluntary $(19.9 \%)$. Medical releases result when 
individuals are determined to be unfit to perform the full range of their duties as a consequence of a health problem. ${ }^{33}$

Military personnel in the CAF have an occupational fitness requirement, one that takes into consideration the potential duties required under the demanding and unpredictable conditions of operational deployments. This requirement is enshrined in the principle of 'Universality of Service', which the CAF enforces by requiring all personnel to be able to perform certain common military tasks and to be deployable to any environment with little medical support. Individuals who are persistently in violation of Universality of Service will be released from service.

When CAF personnel are diagnosed with a medical condition, their clinician determines whether the diagnosis or treatment may interfere with duties. This assessment is a sequential process; it begins with a thorough examination, followed by a comprehensive description of medical employment limitations. Clinicians assign a set of codes (medical categories) that summarise the medical employment limitations' impact on duties. Persistent medical employment limitations and medical categories are validated by physicians in the Medical Standards Section of the CAF, ensuring a standardised application.

While some persistent medical employment limitations will ultimately lead to a release from military service, others will be accommodated. Individuals whose validated limitations are likely to violate Universality of Service undergo an administrative review; those determined to be in violation will ultimately be released.

\section{Covariates of interest}

The primary covariates of interest were: (1) diagnosis era, a proxy for evolutionary enhancements to the CAF mental health system and changes in medical fitness determination practices for individuals with mental disorders, and (2) delay to diagnosis and care, calculated as the latency from most recent Afghanistan-related deployment return date to diagnosis date. The most recent Afghanistan-related deployment return date, prior to a diagnosis, was the most relevant reference point for delay calculations; a majority of the cohort had a single deployment $(80 \%)$ and it is very rare for an individual to deploy with a significant mental health problem. The median date (30 April 2008) categorised diagnosis dates into two eras. This was based on initial investigations that suggested changes in the medically related attrition outcome after 2008. Delay to care was categorised into tertiles: $\leq 245$ days ( $\leq 8$ months), 246636 days ( $8-21$ months) and $\geq 637$ days ( $>21$ months). The delay intervals were chosen based on initial investigations that suggested a non-linear, u-shaped, relationship between delay to care and the outcome.

\section{Potential confounders}

Potential confounders identified in other studies ${ }^{2-17}$ 19-24 included sex, age, element (Army, Navy or Air Force), component (Regular or Reserve Force), rank category, military occupation, years of service, marital status and presence of live-in dependents. Military occupation was categorised into eight groups: ${ }^{34}$ facility support (FS); Health Services (HS); information management; intelligence, surveillance and reconnaissance; operations support (OPS Support); operations technicians; specialist; and operations (OPS), which included combat arms occupations.

Diagnosis-related covariates included indication of a previous history of mental disorder diagnoses and each Diagnostic and Statistical Manual of Mental Disorders, Fourth Edition, Text Revision (DSM-IV-TR) axis, excluding axis IV. Axis I diagnosis codes were categorised into seven groups based on conceptual considerations and disorder patterns: four single diagnosis categories of PTSD, depressive disorder (ie, major depression or dysthymic disorder), adjustment disorder or single 'other' disorder, and three comorbid categories of both PTSD and depressive disorder only, all other comorbid combinations with PTSD and any other non-PTSD comorbid combination. Axis II information was dichotomised for presence of a personality disorder or trait, while axis III information was categorised for presence of a relevant musculoskeletal condition, other condition or none. Functional status (reflected by axis V, the Global Assessment of Functioning) was categorised into four groups: 0-50 (severe symptoms), 51-60 (moderate symptoms), 61-70 (mild symptoms) and 71-100 (transient symptoms).

\section{Data analysis}

The data were analysed using SAS for Windows, V.9.3, and sampling weights accounted for the design effect. Taylor Series Linearization methods ${ }^{35}$ were used to determine $95 \%$ CIs for descriptive statistics. Missing values were identified for marital status, the presence of live-in dependents, and DSM Axis V. The fully conditional specification multiple imputation $\operatorname{method}^{36}$ was implemented when analysing these covariates.

Weighted Kaplan-Meier methods ${ }^{37}$ generated event probabilities. Zero time was defined as the diagnosis date. Event time was the medical release date. Individuals were censored at the earlier of non-medical release date or database extraction date. Ninety-five per cent CIs for Kaplan-Meier estimates were generated using bootstrap methods. ${ }^{38}$

Weighted Cox regression assessed the association of medical release with covariates; results were expressed as HRs and their 95\% CIs, generated using Taylor Series Linearization methods. ${ }^{35}$ The two primary covariates of interest (ie, diagnosis era and delay to care) were forced into a regression model that included potential confounders selected using a two-stage approach. Initially, weighted Cox regressions assessed the unadjusted relationship between each potential confounder and medical release; covariates with a Wald test $\mathrm{p}<0.15$ were included in a multivariate model. In the second stage, 
backwards elimination removed potential confounders using a Wald test $p$ value threshold of 0.05 . Delay to care was handled as an interaction term (delay by whether the diagnosis was Afghanistan deployment-related). This approach optimised the use of available data; among individuals with an Afghanistan deployment-related diagnosis, the Afghanistan-related deployment return date is a relevant proxy for symptom onset timing in delay to care calculations while this is not the case for individuals with diagnoses that are not Afghanistan deploymentrelated. Delay to care HRs were only assessed among those with Afghanistan deployment-related diagnoses. Regression diagnostic plots were reviewed with respect to the proportional hazards assumption. ${ }^{37}$

\section{RESULTS}

\section{Study cohort characteristics}

Medical records were reviewed for 2014 individuals and inaccessible for an additional 31. Of the 2014 individuals, 508 who had a mental disorder diagnosis after the start of their first Afghanistan-related deployment comprised the study sample, representing 5337 individuals in the cohort $(17.5 \%, 95 \%$ CI $16.0 \%$ to $19.0 \%)$.

The study subset consisted largely of men in the Regular Forces of the Army (table 1). At the time of their diagnosis, a majority of individuals were younger than 40, married, had no live-in dependents and had less than 20 years of military service. Although $46.0 \%$ of the study subset were in the 'OPS' occupation category, $79.6 \%$ of these were combat arms occupations. Table 1 summarises the distribution and bivariate associations of these characteristics with medically related attrition.

\section{Mental disorder diagnoses}

Table 2 summarises the distribution of clinical characteristics and their unadjusted association with medically related attrition. A majority of diagnoses $(82.0 \%$; $95 \%$ CI $78.3 \%$ to $85.7 \%)$ were service-related; $77.0 \%$ were attributed to Afghanistan-related deployments and 5.0\% were attributed to other deployments. PTSD was the most diagnosed condition $(49.1 \%$; $95 \%$ CI $44.2 \%$ to $53.9 \%$ ), followed by depressive disorder (ie, major depression or dysthymic disorder; $44.7 \%$; $95 \%$ CI $39.5 \%$ to $49.8 \%)$. PTSD was often a comorbid diagnosis: It occurred alone in $15.7 \%$ (95\% CI $12.1 \%$ to $19.4 \%$ ); with depressive disorder only in $14.3 \%$ (95\% CI $10.9 \%$ to $17.7 \%)$; and with any other disorder(s) in $19.1 \%(95 \%$ CI $15.6 \%$ to $22.5 \%$ ).

The median diagnosis date was 30 April 2008 (range 19 November 2001 to 24 January 2011). The mean delay to care among individuals with an Afghanistan-related mental disorder diagnosis was 551 days (95\% CI 500 to 602; median 400 days).

\section{Medically related attrition}

As of 15 December 2012, 35.5\% (95\% CI 30.8\% to $40.3 \%)$ had released from service, $21.3 \% \quad(95 \%$
CI $17.2 \%$ to $25.5 \%$ ) were medical releases and $14.2 \%$ (95\% CI $10.7 \%$ to $17.8 \%$ ) were for other reasons (ie, $12.7 \%$ voluntary releases; $1.5 \%$ service completion releases). Mean follow-up time from diagnosis to the earlier of release or censor date was 1456 days (95\% CI 1373 to 1538); the median was 1267 days (3.5 years). Individuals who medically released had a mean of 15.5 years of service (95\% CI 13.7 to 17.3 ) at the time of release while those without a medical release had 13.0 years (95\% CI 12.2 to 13.8 ) at censor date.

At 5 years of follow-up, an estimated $25.8 \%$ (95\% CI $20.2 \%$ to $31.4 \%$ ) medically released compared with $13.7 \%$ (95\% CI $10.1 \%$ to $17.3 \%$ ) releasing for other reasons. There was separation in the Kaplan-Meier curves for medical release by diagnosis era (figure 1 ). Follow-up time was limited for recent diagnoses; the maximum was 4.6 years. At 4.5 years of follow-up, an estimated $26.8 \%$ (95\% CI $20.2 \%$ to $33.3 \%$ ) of those diagnosed before 30 April 2008 (median) had medically released compared with $19.7 \% \quad(95 \%$ CI $12.1 \%$ to $27.2 \%)$ thereafter. However, the unadjusted HR comparing diagnosis eras was not statistically significant (HR 1.45 (95\% CI 0.87 to 2.42 ); table 2 ).

At 5 years of follow-up, an estimated $29.1 \%$ (95\% CI $22.5 \%$ to $35.8 \%$ ) of individuals whose mental disorder diagnosis was Afghanistan deployment-related had medically released. Figure 2 displays the Kaplan-Meier curves for medical release as a function of delay to care among individuals with an Afghanistan deployment-related diagnosis. Separation in the curves was again noted: At 5 years of follow-up, an estimated $28.3 \%$ (95\% CI $18.7 \%$ to $37.8 \%$ ) of those with a short delay to care ( $\leq 245$ days) medically released compared with $19.5 \%$ (95\% CI $10.6 \%$ to $28.5 \%$ ) with a medium delay (246636 days) and $46.4 \%$ (95\% CI $27.4 \%$ to $65.4 \%$ ) with a long delay ( $\geq 637$ days). While medical release risk was elevated for individuals having either short or long delays to care, relative to those with medium delays, the unadjusted HR was not statistically significant for short delays (HR 1.69 (95\% CI 0.93 to 3.08)) but it was for long delays (HR 2.24 (95\% CI 1.17 to 4.30 ); table 2).

\section{Cox proportional hazards regression}

The mental disorder history, DSM Axis II, component, sex and marital status potential confounders were discarded during assessment of their unadjusted relationship with medical release; DSM Axis III, element, age category, years of service and live-in dependents were discarded during the backwards elimination regression. The final model that assessed the primary covariates controlled for diagnosis case-mix, military occupation, DSM Axis V (functional impairment) and rank category.

The adjusted model (table 3) showed that individuals diagnosed before the median date of 30 April 2008 had a higher medical release risk relative to those diagnosed recently (adjusted HR (aHR) 1.77 (95\% CI 1.01 to 3.11)). Among individuals with an Afghanistan deployment-related mental disorder, those with a long 
Table 1 Demographic and military characteristics of the study subset (weighted $N=5337$; sample $n=508$ ) and their unadjusted association with medically related attrition

\begin{tabular}{|c|c|c|c|c|c|c|}
\hline \multirow[b]{2}{*}{ Characteristic } & \multirow{2}{*}{$\begin{array}{l}\text { Unweighted } \\
\text { Sample } \\
\text { number }\end{array}$} & \multirow[b]{2}{*}{ Weighted (\%) } & \multicolumn{2}{|c|}{ Medical release } & \multirow{2}{*}{$\begin{array}{l}\text { Unadjusted } \\
\text { HR }\end{array}$} & \multirow[b]{2}{*}{$95 \% \mathrm{Cl}$} \\
\hline & & & Per cent & $95 \% \mathrm{Cl}$ & & \\
\hline \multicolumn{7}{|l|}{ Occupation categorisation* } \\
\hline FS & 12 & 2.7 & 54.8 & 24.4 to 85.3 & $5.36^{\mathrm{a}}$ & 2.21 to 12.98 \\
\hline $\mathrm{HS}$ & 28 & 4.8 & 12.6 & 0.9 to 24.3 & 0.76 & 0.26 to 2.26 \\
\hline IM & 31 & 4.8 & 18.2 & 5.0 to 31.5 & 0.79 & 0.32 to 2.00 \\
\hline ISR & 22 & 3.5 & 21.3 & 4.2 to 38.5 & 1.29 & 0.57 to 2.92 \\
\hline OPS Support & 96 & 15.4 & 29.9 & 19.0 to 40.8 & $1.75^{\mathrm{c}}$ & 0.98 to 3.12 \\
\hline OPS Tech & 58 & 16.0 & 21.2 & 9.3 to 33.1 & 0.81 & 0.36 to 1.80 \\
\hline Specialist & 34 & 6.8 & 15.7 & 2.7 to 28.6 & 0.86 & 0.34 to 2.18 \\
\hline OPS & 227 & 46.0 & 18.6 & 12.7 to 24.5 & Reference & Reference \\
\hline \multicolumn{7}{|c|}{ Occupation categorisation (aggregated)* } \\
\hline FS & 12 & 2.7 & 54.8 & 24.4 to 85.3 & $5.75^{\mathrm{a}}$ & 2.46 to 13.46 \\
\hline OPS Support & 96 & 15.4 & 29.9 & 19.0 to 40.8 & $1.88^{\mathrm{b}}$ & 1.11 to 3.20 \\
\hline Other & 400 & 81.9 & 18.6 & 14.2 to 23.0 & Reference & Reference \\
\hline \multicolumn{7}{|l|}{ Component } \\
\hline Reserve Forces & 23 & 6.7 & 12.7 & 0 to 28.7 & 0.68 & 0.16 to 2.81 \\
\hline Regular Forces & 485 & 93.3 & 21.9 & 17.6 to 26.2 & Reference & Reference \\
\hline \multicolumn{7}{|l|}{ Element ${ }^{\star}$} \\
\hline Air Force & 66 & 12.9 & 26.9 & 13.9 to 39.9 & $2.21^{\mathrm{c}}$ & 0.94 to 5.22 \\
\hline Navy & 65 & 21.0 & 17.4 & 7.3 to 27.5 & Reference & Reference \\
\hline Army & 377 & 66.1 & 21.5 & 16.8 to 26.2 & $1.84^{\mathrm{C}}$ & 0.95 to 3.57 \\
\hline \multicolumn{7}{|l|}{ Rank* $^{*}$} \\
\hline Officer & 48 & 10.4 & 4.9 & 0 to 9.8 & Reference & Reference \\
\hline SNCM & 130 & 24.8 & 25.4 & 16.3 to 34.5 & $6.10^{\mathrm{a}}$ & 1.92 to 19.33 \\
\hline JNCM & 330 & 64.7 & 22.4 & 17.2 to 27.6 & $4.63^{\mathrm{a}}$ & 1.51 to 14.17 \\
\hline \multicolumn{7}{|l|}{ Sex } \\
\hline Female & 62 & 9.8 & 25.2 & 14.5 to 36.0 & 1.25 & 0.74 to 2.11 \\
\hline Male & 446 & 90.2 & 20.9 & 16.4 to 25.4 & Reference & Reference \\
\hline \multicolumn{7}{|l|}{$\mathrm{Age}^{*}$} \\
\hline$\leq 29$ & 140 & 27.9 & 11.2 & 5.5 to 16.8 & Reference & Reference \\
\hline $30-39$ & 234 & 44.5 & 24.7 & 18.3 to 31.1 & $1.85^{\mathrm{c}}$ & 0.98 to 3.47 \\
\hline$\geq 40$ & 134 & 27.6 & 26.1 & 17.4 to 34.8 & $2.38^{\mathrm{b}}$ & 1.20 to 4.72 \\
\hline \multicolumn{7}{|l|}{ Years of service ${ }^{*}$} \\
\hline$\leq 9$ & 214 & 43.1 & 16.3 & 10.7 to 22.0 & Reference & Reference \\
\hline 10-19 & 194 & 35.5 & 24.6 & 17.5 to 31.6 & 1.42 & 0.86 to 2.35 \\
\hline$\geq 20$ & 100 & 21.4 & 26.0 & 16.0 to 35.9 & $1.84^{\mathrm{b}}$ & 1.01 to 3.37 \\
\hline \multicolumn{7}{|l|}{ Marital status $(\mathrm{MI}) \dagger$} \\
\hline Married & 350 & 68.7 & 23.1 & 17.8 to 28.3 & Reference & Reference \\
\hline Single-never married & 100 & 20.0 & 14.5 & 7.5 to 21.4 & $0.58^{\mathrm{c}}$ & 0.32 to 1.06 \\
\hline Divorced/widowed/separated & 49 & 9.7 & 17.5 & 5.9 to 29.1 & & \\
\hline Unspecified & 9 & 1.6 & 55.3 & 18.7 to 92.0 & 0.70 & 0.33 to 1.49 \\
\hline \multicolumn{7}{|l|}{ Live-in dependents $(\mathrm{Ml})^{\star}+$} \\
\hline Yes & 232 & 42.8 & 27.3 & 20.4 to 34.2 & $1.61^{\mathrm{b}}$ & 1.03 to 2.53 \\
\hline No & 246 & 50.8 & 16.5 & 11.4 to 21.6 & Reference & Reference \\
\hline Unspecified & 30 & 6.4 & 19.5 & 3.5 to 35.5 & & \\
\hline
\end{tabular}

Otherwise, $p>0.15$.

*The Wald test $p$ value was less than 0.15 for variables: occupation categorisation, element, rank, age, years of service and live-in dependents (MI).

†MI was used to compute the unadjusted HR for variables: marital status (MI) and live-in dependents (MI).

${ }^{a} p \leq 0.01$.

${ }_{\mathrm{b}}^{\mathrm{p}} \leq 0.05$.

$\mathrm{c} \leq 0.1$.

$\mathrm{d}_{\mathrm{p}} \leq 0.15$.

FS, facility support; HS, health services; IM, information management; ISR, intelligence, surveillance and reconnaissance; JNCM, junior non-commissioned member; MI, multiple imputation used; OPS Support, operations support; OPS Tech, operations technicians; OPS, operations; SNCM, senior non-commissioned member. 


\begin{tabular}{|c|c|c|c|c|c|c|}
\hline \multirow[b]{2}{*}{ Clinical characteristic } & \multirow{2}{*}{$\begin{array}{l}\text { Unweighted } \\
\text { Sample number }\end{array}$} & \multirow[b]{2}{*}{ Weighted (\%) } & \multicolumn{2}{|c|}{ Medical release } & \multirow[b]{2}{*}{ Unadjusted HR } & \multirow[b]{2}{*}{ (95\% Cl) } \\
\hline & & & Per cent & $95 \% \mathrm{Cl}$ & & \\
\hline \multicolumn{7}{|l|}{ Diagnosis timing* (median split) } \\
\hline Before 30 April 2008 & 235 & 49.8 & 30.6 & 23.9 to 37.4 & $1.45^{\mathrm{e}}$ & 0.87 to 2.42 \\
\hline \multicolumn{7}{|l|}{ Delay to care ${ }^{*}$, (tertile split) } \\
\hline Short: $\leq 245$ days & 138 & 33.0 & 27.3 & 19.4 to 35.2 & $1.69^{d}$ & 0.93 to 3.08 \\
\hline Medium: 246-636 days & 144 & 33.9 & 14.7 & 8.4 to 20.9 & Reference & Reference \\
\hline Yes & 142 & 28.4 & 23.0 & 15.1 to 30.8 & 0.84 & 0.52 to 1.37 \\
\hline None indicated & 366 & 71.6 & 20.7 & 15.8 to 25.6 & Reference & Reference \\
\hline \multicolumn{7}{|l|}{ Mental disorder diagnoses } \\
\hline Any PTSD & 272 & 49.1 & 28.9 & 22.8 to 35.0 & $2.22^{\mathrm{b}}$ & 1.36 to 3.63 \\
\hline No PTSD & 236 & 50.9 & 14.0 & 8.6 to 19.4 & Reference & Reference \\
\hline Any non-PTSD anxiety & 128 & 26.8 & 14.7 & 7.9 to 21.6 & $0.54^{\mathrm{c}}$ & 0.31 to 0.95 \\
\hline No non-PTSD anxiety & 380 & 73.2 & 23.7 & 18.7 to 28.8 & Reference & Reference \\
\hline No bipolar disorder & 502 & 98.3 & 21.1 & 16.9 to 25.2 & Reference & Reference \\
\hline Any other mood disorder & 7 & 1.7 & 9.8 & 0 to 28.7 & 0.25 & 0.03 to 2.30 \\
\hline No other mood disorder & 501 & 98.3 & 21.5 & 17.3 to 25.7 & Reference & Reference \\
\hline Any somatoform disorder & 6 & 1.5 & 79.9 & 44.5 to 100 & $4.52^{\mathrm{c}}$ & 1.16 to 17.64 \\
\hline No somatoform disorder & 502 & 98.5 & 20.4 & 16.3 to 24.6 & Reference & Reference \\
\hline Any substance abuse disorder & 83 & 16.1 & 19.3 & 9.7 to 28.9 & 0.93 & 0.52 to 1.66 \\
\hline No substance abuse disorder & 425 & 83.9 & 21.7 & 17.1 to 26.3 & Reference & Reference \\
\hline \multicolumn{7}{|l|}{ Mental disorder diagnosis case-mix ${ }^{*}$} \\
\hline PTSD only & 79 & 15.7 & 25.5 & 13.8 to 37.2 & 1.50 & 0.58 to 3.88 \\
\hline Depressive disorder only & 58 & 13.4 & 20.4 & 7.1 to 33.7 & 1.46 & 0.51 to 4.20 \\
\hline Other single diagnosis only & 47 & 10.8 & 4.0 & 0.0 to 8.4 & $0.21^{\mathrm{c}}$ & 0.05 to 0.87 \\
\hline Adjustment disorder only & 59 & 10.7 & 18.2 & 5.6 to 30.7 & Reference & Reference \\
\hline PTSD and depressive disorder & 80 & 14.3 & 33.8 & 22.5 to 45.2 & $2.38^{\mathrm{d}}$ & 0.97 to 5.82 \\
\hline PTSD and other & 113 & 19.1 & 28.0 & 18.7 to 37.3 & 1.82 & 0.75 to 4.41 \\
\hline Any other combination (excluding PTSD) & 72 & 15.9 & 12.7 & 3.7 to 21.7 & 0.74 & 0.25 to 2.22 \\
\hline
\end{tabular}




\section{Table 2 Continued}

\begin{tabular}{|c|c|c|c|c|c|c|}
\hline \multirow[b]{2}{*}{ Clinical characteristic } & \multirow[b]{2}{*}{$\begin{array}{l}\text { Unweighted } \\
\text { Sample number }\end{array}$} & \multirow[b]{2}{*}{ Weighted (\%) } & \multicolumn{2}{|c|}{ Medical release } & \multirow[b]{2}{*}{ Unadjusted HR } & \multirow[b]{2}{*}{$(95 \% \mathrm{Cl})$} \\
\hline & & & Per cent & $95 \% \mathrm{Cl}$ & & \\
\hline \multicolumn{7}{|c|}{ DSM-IV_-Axis III general medical conditions present* } \\
\hline Musculoskeletal & 126 & 24.6 & 31.2 & 21.7 to 40.6 & $1.92^{\mathrm{c}}$ & 1.15 to 3.21 \\
\hline Other & 117 & 22.5 & 22.8 & 13.5 to 32.0 & 1.42 & 0.80 to 2.53 \\
\hline None indicated & 265 & 52.9 & 16.1 & 11.1 to 21.1 & Reference & Reference \\
\hline \multicolumn{7}{|c|}{ DSM-IV—Axis V global assessment of functioning (MI) ${ }^{\star} \ddagger$} \\
\hline 0-50: Severe symptoms & 46 & 7.5 & 42.5 & 27.6 to 57.4 & $9.32^{\mathrm{b}}$ & 2.89 to 30.09 \\
\hline 51-60: Moderate symptoms & 152 & 27.8 & 19.7 & 13.1 to 26.4 & $2.98^{d}$ & 0.89 to 9.95 \\
\hline 61-70: Mild symptoms & 163 & 33.7 & 25.3 & 17.0 to 33.6 & $3.76^{\mathrm{c}}$ & 1.23 to 11.49 \\
\hline 71-100: Transient symptoms & 55 & 11.7 & 7.1 & 1.8 to 12.3 & Reference & Reference \\
\hline Unspecified & 92 & 19.3 & 17.0 & 7.6 to 26.5 & & \\
\hline
\end{tabular}

Otherwise, $p>0.15$

${ }^{*}$ The Wald test $p$ value was less than 0.15 for variables: diagnosis timing, delay to care, mental disorder diagnosis case-mix (including any PTSD, any non-PTSD anxiety disorder, any depressive disorder and any somatoform disorder), DSM-IV-Axis III general medical conditions present and DSM-IV-Axis V global assessment of functioning (MI).

tDelay to care is calculated as the latency from most recent Afghanistan-related deployment return date to diagnosis date, for diagnoses attributed to an Afghanistan deployment ( $\mathrm{n}=415$ ). $\ddagger$ MI was used to compute the unadjusted HR for variable: DSM-IV-Axis V global assessment of functioning (MI).

$\mathrm{b}_{\mathrm{p}} \leq 0.01$

${ }_{p} \leq 0.05$

$\mathrm{d} \leq 0.1$.

${ }^{\mathrm{e}} \mathrm{p} \leq 0.15$

DSM-IV, Diagnostic and Statistical Manual of Mental Disorders, Fourth Edition; MI, multiple imputation used; PTSD, post-traumatic stress disorder. 


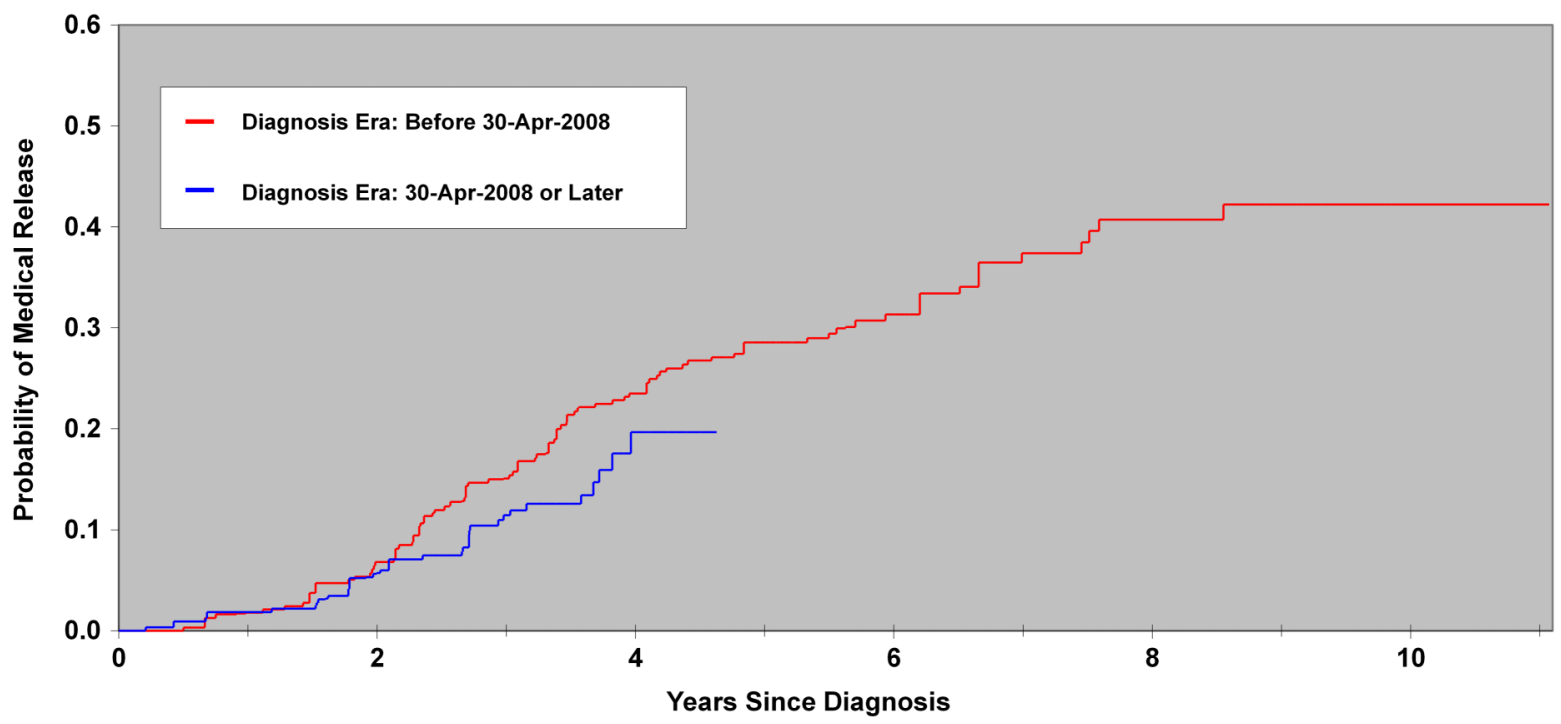

Figure 1 Kaplan-Meier estimates of the probability of a medical release from military service with increasing time from mental disorder diagnosis by diagnosis era. The diagnosis era was defined using a median split: before 30 April 2008 (sample $n=235$, weighted $\mathrm{N}=2659$ ) and 30 April 2008 or later (sample $\mathrm{n}=273$, weighted $\mathrm{N}=2678$ ).

delay to care had a higher medical release risk relative to those with a medium delay (aHR 2.47 (95\% CI 1.28 to 4.76)); there was no statistically significant difference in risk between those with short and intermediate delays to care (aHR 1.55 (95\% CI 0.85 to 2.83 )).

Medically related attrition risk varied by diagnosis mix (table 3). Individuals with both a PTSD and depression diagnosis had a higher risk relative to those with adjustment disorder alone (aHR 2.88 (95\% CI 1.05 to 7.88)). However, while individuals with PTSD alone, depression alone or PTSD with other mental disorder diagnoses had comparably elevated medical release risks relative to those with adjustment disorder alone, these were not statistically significant. The medically related attrition risk was also higher for individuals in the axis $\mathrm{V}$ severe symptoms category (aHR 8.48 (95\% CI 1.86 to 38.73)) relative to those with transient symptoms and elevated but not statistically significant for individuals with moderate or mild symptoms. Additionally, medically related attrition risk was higher for individuals in both the

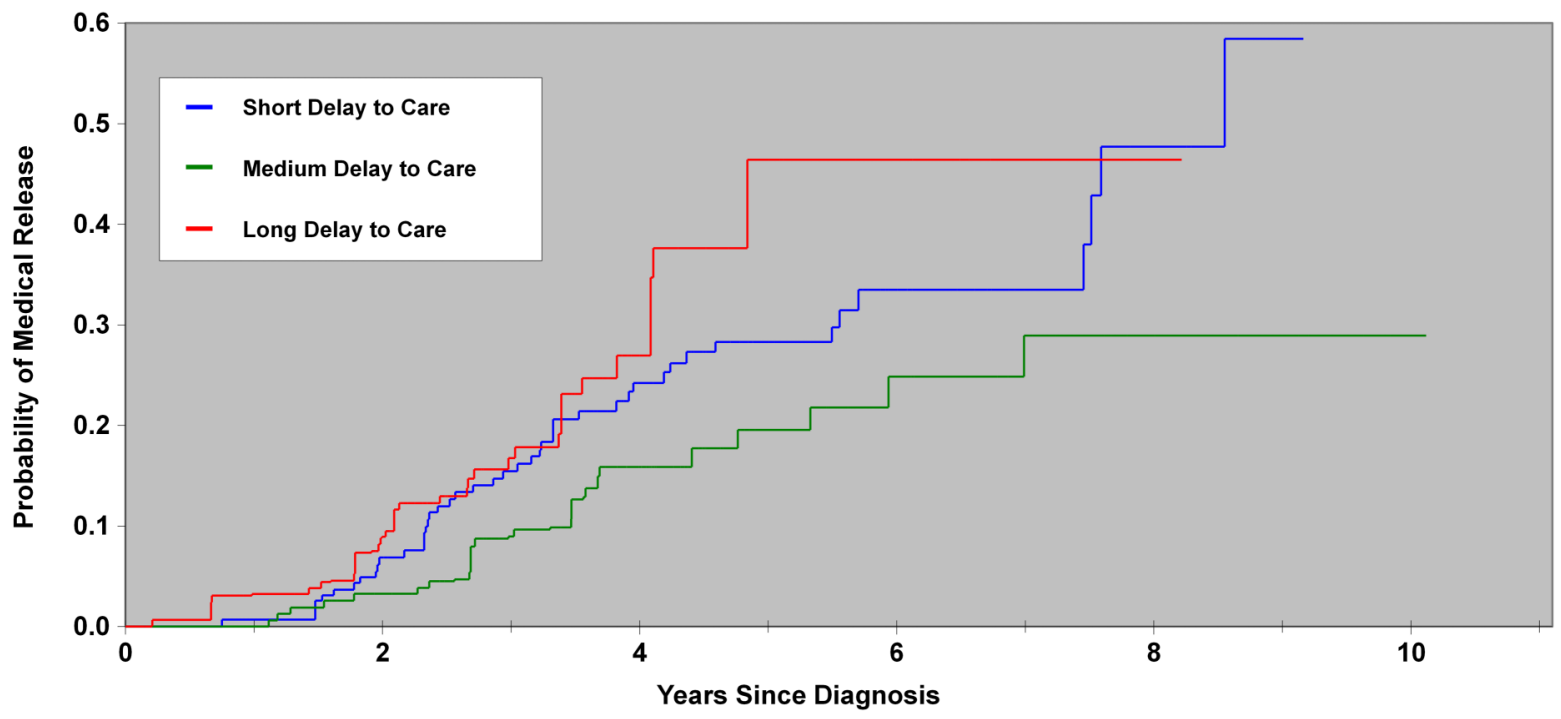

Figure 2 Kaplan-Meier estimates of the probability of a medical release from military service with increasing time from a mental disorder diagnosis by delay to care. Delay was calculated from the most recent Afghanistan deployment return date to the diagnosis date of an Afghanistan deployment-related disorder. Delays to care were categorised into tertiles: short ( $\leq 245$ days; sample $n=138$, weighted $N=1355$ ), medium (246-636 days; sample $n=144$, weighted $N=1394$ ) and long ( $\geq 637$ days; sample $\mathrm{n}=133$, weighted $\mathrm{N}=1360$ ). 
Table 3 Cox proportional hazard regression assessment of the adjusted association of demographic, military and clinical characteristics with medical release, following a backwards elimination selection process*

\begin{tabular}{|c|c|c|}
\hline Characteristic & Adjusted HR & $95 \% \mathrm{Cl}$ \\
\hline \multicolumn{3}{|l|}{ Diagnosis Timing (median split) } \\
\hline 30 April 2008 or later & Reference & Reference \\
\hline Before 30 April 2008 & $1.77^{\mathrm{d}}$ & 1.01 to 3.11 \\
\hline \multicolumn{3}{|l|}{ Delay to care (tertile split) } \\
\hline Short: $\leq 245$ days & 1.55 & 0.85 to 2.83 \\
\hline Medium: 246-636 days & Reference & Reference \\
\hline Long: $\geq 637$ days & $2.47^{\mathrm{C}}$ & 1.28 to 4.76 \\
\hline \multicolumn{3}{|l|}{ Mental disorder diagnosis case-mix } \\
\hline PTSD only & 2.17 & 0.77 to 6.07 \\
\hline Depressive disorder only & 2.29 & 0.68 to 7.65 \\
\hline Other single diagnosis only & 0.32 & 0.07 to 1.48 \\
\hline Adjustment disorder only & Reference & Reference \\
\hline PTSD and depressive disorder & $2.88^{d}$ & 1.05 to 7.88 \\
\hline PTSD and other & 1.99 & 0.72 to 5.48 \\
\hline Any other combination (excluding PTSD) & 1.05 & 0.29 to 3.78 \\
\hline \multicolumn{3}{|c|}{ DSM IV-Axis V global assessment of functioning (MI) } \\
\hline 0-50: Severe symptoms & $8.48^{c}$ & 1.86 to 38.73 \\
\hline 51-60: Moderate symptoms & 2.44 & 0.52 to 11.53 \\
\hline 61-70: Some mild symptoms & $3.45^{\mathrm{e}}$ & 0.81 to 14.65 \\
\hline 71-100: Transient symptoms & Reference & Reference \\
\hline \multicolumn{3}{|l|}{ Occupation categorisation (aggregated) } \\
\hline FS & $4.98^{\mathrm{C}}$ & 1.62 to 15.32 \\
\hline OPS Support & $2.40^{\mathrm{C}}$ & 1.41 to 4.08 \\
\hline Other & Reference & Reference \\
\hline \multicolumn{3}{|l|}{ Rank } \\
\hline Officer & Reference & Reference \\
\hline SNCM & $4.93^{\mathrm{d}}$ & 1.35 to 18.05 \\
\hline JNCM & $4.12^{d}$ & 1.15 to 14.73 \\
\hline
\end{tabular}

Otherwise, $p>0.10$.

*A backwards elimination selection process identified the following variables for removal from the final assessment model, listed in their order of removal: live-in dependents, years of service, element, DSM IV-Axis III and age.

†Delay to care is calculated as the latency from most recent Afghanistan-related deployment return date to diagnosis date, for diagnoses attributed to an Afghanistan deployment $(n=415)$.

${ }^{c} \mathrm{p} \leq 0.01$.

$\mathrm{d} p \leq 0.05$.

$e^{e} \leq 0.10$.

DSM-IV, Diagnostic and Statistical Manual of Mental Disorders; FS, facility support; JNCM, junior non-commissioned member; MI, multiple imputation used; OPS Support, Operations Support; PTSD, post-traumatic stress disorder; SNCM, Senior non-commissioned member.

junior (aHR 4.12 (95\% CI 1.15 to 14.73 )) and senior (aHR 4.93 (95\% CI 1.35 to 18.05$)$ ) non-commissioned member rank categories relative to officers.

The occupation categorisation was modified following the assessment of its unadjusted relationship with medical release, keeping FS and OPS Support, which had elevated HRs, but aggregating other occupational categories, which did not. Attrition risk was higher for the FS (aHR 4.98 (95\% CI 1.62 to 15.32)) and OPS Support (aHR 2.40 (95\% CI 1.41 to 4.08 )) categories relative to the aggregated occupational category. FS included occupations such as construction engineers, fire fighters, plumber/gas fitter and technicians in: water, fuels and the environment, electrical distribution, plumbing and heating, refrigeration and mechanical systems, and weather systems processing. OPS Support included occupations such as logistics support, air traffic controllers, supply technicians, traffic technicians, postal clerks, administrative clerks, financial clerks, resource management support clerks and mobile support equipment operators.

\section{DISCUSSION}

\section{Key findings}

Our study's primary objective was to explore the association of mental disorder diagnosis era and delay to care with medically related attrition. We observed that an unfavourable occupational prognosis was more likely for individuals diagnosed before 30 April 2008 (aHR=1.77). Also as hypothesised, those with the longest delay to care ( $>21$ months) had a higher risk of medical release relative to those with an intermediate delay $(\mathrm{aHR}=2.47$ ). There was no significant difference in outcome between those with short and intermediate delays to care.

We identified several other independent risk factors for medically related attrition, including disorder severity, diagnosis mix (specifically, PTSD comorbid with a 
depressive disorder), being a non-commissioned member, and being in certain occupational categories. A number of potential risk factors had no significant relationship with the primary outcome, including DSM Axis II comorbidity, complicating musculoskeletal or other medical problems, a history of mental disorders, age, years of service, sex, marital status, live-in dependents, component (Regular or Reserve Forces), and element (Army, Navy or Air Force).

\section{Comparison with other findings}

We could not identify other studies that compared military occupational outcome from one time period to another among individuals with mental disorders. While there is evidence that PTSD remission rates have been increasing over time, ${ }^{39}$ the effect on attrition is unknown. Studies on the association of prognosis relative to the timing of care are sparse, though consistent with our findings, Maguen $e t a \hat{l}^{22}$ found indications that veterans who initiated PTSD treatment earlier after deployment return were more likely to see symptom improvement.

Our finding of a less favourable occupational outcome among individuals with more severe illness and in lower ranks mirrors the unadjusted findings from others. ${ }^{17} 2324$ In contrast, while we observed a less favourable outcome for certain occupations, these occupations differed from ones identified by others (combat arms occupations). ${ }^{17} \quad 24$ Other studies identified gender, ${ }^{17}$ service length ${ }^{17}{ }^{24}$ and diagnosis ${ }^{17} 2324$ to be associated with outcomes in their adjusted models. We did not find significant associations for gender or service length in our adjusted models but we did for diagnosis mix. However, these other studies were among different populations, used premature release from service $^{1724}$ or return to occupational fitness ${ }^{23}$ as outcomes and they emanate from other military organisations in which these and other prognostic factors may play out differently.

\section{Strengths and limitations}

This study's primary strength is that it explores an important but under-researched issue: the effects of mental health services renewal and delay to care on occupational prognosis of military mental health problems. We were able to control for a broad range of potential confounders, notably severity and various forms of medical and psychiatric comorbidity. The time-to-event analytic approach allowed for an efficient use of the data.

This study's primary limitation is its observational nature: We sought to explore changes in prognosis as a function of diagnosis era, itself a proxy for evolutionary changes in the CAF mental health system. It is possible that unmeasured factors could account for the observed improvement in prognosis. We were unable to identify precise implementation time points for the many concurrent changes, precluding identification of the drivers of the more favourable outcomes seen over time. We could not control for unmeasured factors related to careseeking behaviours and the timing of first care which are potentially associated with occupational prognosis.

It was not possible to distinguish medically related attrition that was due to physical issues from those that were due to mental health issues for the entire study population. Augmented data on the medical condition attributed to a medical release began to be collected in 2009 and, therefore, was available for only a subset of the individuals in this study (ie, $46 \%$ of those with a medical release). Among these individuals with such data and a medical release, $56 \%$ had a mental disorder identified as the primary condition attributed to the medical release; an additional $3 \%$ had a mental disorder identified as a secondary condition.

We calculated delay to care using the most recent Afghanistan-related deployment return date prior to diagnosis and not the unknown timing of the precipitating incident or symptom onset. While our sample size was large enough to identify at least some significant predictors (notably those related to our primary objective), there was limited power to detect differences for some comparisons. Finally, there is a possibility that our favourable findings reflect a lengthening of the delay to medically related attrition, a beneficial short-term outcome, as opposed to a shift from releases for medical reasons to releases for other reasons (eg, term of service completed), a beneficial long-term outcome.

\section{Implications}

A sizable fraction of personnel who deployed in support of the CAF mission in Afghanistan will be diagnosed with a service-related mental disorder and we observed that almost $30 \%$ of those who do will medically release within 5 years. Those who medically release will take their substantial military experience ( 15.5 years, on average) with them when they leave. This speaks to the sizable impact of service-related mental disorders on military organisations and to the benefits of efforts to reduce medically related attrition.

We found encouraging evidence that occupational prognosis for postdeployment mental disorders improved significantly over the past 12 years in the CAF. Since many possible sources of confounding were addressed, we believe that this finding is related to mental health system changes. Further research may permit identification of the relative contribution of each of the many implemented changes.

We also found that those who presented for care relatively late ( $>21$ months after return) had poorer occupational prognosis. However, while prognosis tended to be poorer for individuals with a short delay to care relative to those with a medium delay, this did not achieve statistical significance. The decision to seek care is a complicated one. Illness perceptions and concerns such as stigma, career impact of care-seeking, eligibility for later benefits, confidence in care provision, uncertainty of 
need, etc, can influence care-seeking; ${ }^{26}$ we did not measure these factors. Assessment of these would be helpful in future research. While our findings on delay to care and prognosis are encouraging, definitive studies are needed to assess specific interventions to shorten the delay to care (eg, antistigma programmes, screening).

Decreases in delay to care were an explicit target of CAF mental health services renewal, and we found that shorter delays were independently associated with more favourable outcomes. Hence, if these promotion activities were successful, our estimate of the beneficial effect of mental health services renewal (captured by diagnosis era) may be conservative.

Some of our findings may help clinicians prognosticate more effectively, at least with occupational prognosis. Interestingly, a history of mental health problems, medical comorbidity, axis II comorbidity and axis I comorbidity patterns other than PTSD comorbid with depressive disorder were not associated with an unfavourable occupational outcome.

In contrast, non-commissioned members had a fourfold greater risk of medical release relative to officers. Those in two occupational categories also had an elevated risk. This is surprising, given that the mental health-related fitness standards largely do not differ by rank or occupation. We have no clear explanation for these phenomena.

\section{CONCLUSION}

The CAF and other military organisations have invested heavily in their mental health systems. We found that these investments were associated with an encouraging decrease in medically related attrition and that shorter delays to care may also lead to better occupational outcomes. These findings speak to the potential impact of mental health services renewal and efforts to shorten delay to care. The high prevalence of deploymentrelated mental disorders, their sizeable risk for medically related attrition and the substantial expertise inevitably lost during medical release all reinforce the potential impact of these efforts.

\section{Author affiliations \\ ${ }^{1}$ Directorate of Mental Health, Canadian Forces Health Services Group Headquarters, Ottawa, Ontario, Canada \\ ${ }^{2}$ Faculty of Medicine, Department of Family Medicine, University of Ottawa, Ottawa, Ontario, Canada}

Acknowledgements The authors thank Julie Lanouette and Suzanne Giroux for their assistance with the data collection from patient medical records.

Contributors DB had full access to all data in the study and takes responsibility for the integrity of the data and accuracy of the data analysis. Both DB and MAZ contributed to the study design, the interpretation of the study results as well as the writing and revising of the manuscript. Both DB and MAZ have read and agree with the manuscript's final content.

Funding This work was supported by funding from the Canadian Armed Forces Surgeon General's Medical Research Program.

Competing interests None declared.
Ethics approval This research was approved by Veritas Research Ethics Board (Dorval, QC).

Provenance and peer review Not commissioned; externally peer reviewed.

Data sharing statement No additional data are available.

Open Access This is an Open Access article distributed in accordance with the Creative Commons Attribution Non Commercial (CC BY-NC 4.0) license, which permits others to distribute, remix, adapt, build upon this work noncommercially, and license their derivative works on different terms, provided the original work is properly cited and the use is non-commercial. See: http:// creativecommons.org/licenses/by-nc/4.0/

\section{REFERENCES}

1. Seal KH, Metzler TJ, Gima KS, et al. Trends and risk factors for mental health diagnoses among Iraq and Afghanistan veterans using Department of Veterans Affairs health care, 2002-2008. Am J Public Health 2009:99:1651-8.

2. Boulos D, Zamorski MA. Deployment-related mental disorders among Canadian Forces personnel deployed in support of the mission in Afghanistan, 2001-2008. CMAJ 2013;185:E545-52.

3. Ireland RR, Kress AM, Frost LZ. Association between mental health conditions diagnosed during initial eligibility for military health care benefits and subsequent deployment, attrition, and death by suicide among active duty service members. Mil Med 2012;177:1149-56.

4. Schmied EA, Highfill-McRoy RM, Larson GE. Mental health and turnover following an initial term of military service. Mil Med 2012;177:766-72.

5. Buckman JE, Forbes HJ, Clayton T, et al. Early service leavers: a study of the factors associated with premature separation from the UK Armed Forces and the mental health of those that leave early. Eur J Public Health 2013;23:410-15.

6. Sikorski C, Emerson MA, Cowan DN, et al. Risk factors for medical disability in U.S. enlisted Marines: fiscal years 2001 to 2009 . Mil Med 2012;177:128-34.

7. Crain JA, Larson GE, Highfill-McRoy RM, et al. Postcombat outcomes among Marines with preexisting mental diagnoses. J Trauma Stress 2011;24:671-9.

8. Niebuhr DW, Krampf RL, Mayo JA, et al. Risk factors for disability retirement among healthy adults joining the U.S. Army. Mil Med 2011:176:170-5.

9. Garvey Wilson AL, Messer SC, Hoge CW. U.S. military mental health care utilization and attrition prior to the wars in Iraq and Afghanistan. Soc Psychiatry Psychiatr Epidemiol 2009;44: 473-81.

10. Bell NS, Schwartz CE, Harford TC, et al. Temporal changes in the nature of disability: U.S. Army soldiers discharged with disability, 1981-2005. Disabil Health J 2008;1:163-71.

11. Creamer M, Carboon I, Forbes AB, et al. Psychiatric disorder and separation from military service: a 10-year retrospective study. $\mathrm{Am} \mathrm{J}$ Psychiatry 2006;163:733-4.

12. Rona RJ, Hooper R, Greenberg N, et al. Medical downgrading, self-perception of health, and psychological symptoms in the British Armed Forces. Occup Environ Med 2006;63:250-4.

13. Hoge CW, Auchterlonie JL, Milliken CS. Mental health problems, use of mental health services, and attrition from military service after returning from deployment to Iraq or Afghanistan. JAMA 2006;295:1023-32.

14. Hoge CW, Toboni HE, Messer SC, et al. The occupational burden of mental disorders in the U.S. military: psychiatric hospitalizations, involuntary separations, and disability. Am J Psychiatry 2005;162:585-91.

15. Cigrang JA, Carbone EG, Todd S, et al. Mental health attrition from Air Force basic military training. Mil Med 1998;163:834-8.

16. Patzkowski JC, Rivera JC, Ficke JR, et al. The changing face of disability in the US Army: the Operation Enduring Freedom and Operation Iraqi Freedom effect. J Am Acad Orthop Surg 2012;20 (Suppl 1):S23-30.

17. Jones N, Fear NT, Greenberg N, et al. Occupational outcomes in soldiers hospitalized with mental health problems. Occup Med (Lond) 2009;59:459-65.

18. The Conference Board of Canada. Mental health issues in the labour force: reducing the economic impact on Canada. http://www. conferenceboard.ca/e-library/abstract.aspx?did=4957 (accessed 12 Sep 2014).

19. Packnett ER, Gubata ME, Cowan DN, et al. Temporal trends in the epidemiology of disabilities related to posttraumatic stress disorder in 
the U.S. Army and Marine Corps from 2005-2010. J Trauma Stress 2012;25:485-93.

20. Bell NS, Schwartz CE, Harford T, et al. The changing profile of disability in the U.S. Army: 1981-2005. Disabil Health J 2008;1:14-24.

21. Iversen A, Nikolaou V, Greenberg N, et al. What happens to British veterans when they leave the armed forces? Eur J Public Health 2005;15:175-84.

22. Swedler DI, Knapik JJ, Williams KW, et al. Risk factors for medical discharge from United States Army basic combat training. Mil Med 2011;176:1104-10.

23. Flynn CF, McGlohn S, Miles RE. Occupational outcome in military aviators after psychiatric hospitalization. Aviat Space Environ Med 1996;67:8-13.

24. Jones N, Fear NT, Jones M, et al. Long-term military work outcomes in soldiers who become mental health casualties when deployed on operations. Psychiatry 2010;73:352-64.

25. The Department of National Defence and The Canadian Armed Forces. Surgeon General's mental health strategy: Canadian Forces Health Services Group-an evolution of excellence. http://cmp-cpm. forces.mil.ca/health-sante/pub/pdf/sgmhs-smgmsm-eng.pdf (accessed 03 Oct2013).

26. Gould M, Adler A, Zamorski M, et al. Do stigma and other perceived barriers to mental health care differ across armed forces? J $R$ Soc Med 2010;103:148-56.

27. Department of National Defence. Operational stress injury social support program web site. http://www.osiss.ca (accessed 19 Sep 2014).

28. Canadian Armed Forces. Road to mental readiness (R2MR) program. http://www.forces.gc.ca/en/caf-community-health-servicesr2mr/index.page (accessed 12 Sep 2014).

29. Commonwealth of Australian. Capability through mental fitness: 2011 Australian Defence Force mental health and wellbeing strategy. http://www.defence.gov.au/health/dmh/docs/2011ADF MentalHealthandWellbeingStrategy.pdf (accessed 29 Sep 2014)

30. Department of Defence Task Force on Mental Health. An achievable vision: report of the Department of Defense task force on mental health. http://www.health.mil/ /media/MHS/Report\%20Files/ MHTFReportFinal.ashx (accessed 29 Sep 2014).

31. Hoge CW, Lesikar SE, Guevara R, et al. Mental disorders among U. S. military personnel in the 1990s: association with high levels of health care utilization and early military attrition. Am J Psychiatry 2002;159:1576-83.

32. Maguen S, Madden E, Neylan TC, et al. Timing of mental health treatment and PTSD symptom improvement among Iraq and Afghanistan veterans. Psychiatr Serv 2014;65:1414-19.

33. The Department of National Defence and The Canadian Armed Forces. Queen's regulations and orders for the Canadian Forceschapter 15 release. http://www.admfincs.forces.gc.ca/qro-orf/vol-01/ doc/chapter-chapitre-015.pdf (accessed 27 Jun 2014).

34. Bender P. Towards a sustainable CF: a risk analysis model. Technical Memorandum DRDC CORA TM 2005-10. Ottawa: Department of National Defence (Canada), 2005.

35. Wolter KM. Introduction to variance estimation. New York, NY: Springer-Verlag, 1985.

36. van Buuren S. Multiple imputation of continuous and discrete data by fully conditional specification. Stat Methods Med Res 2007; 16:219-42.

37. Klein JP, Moeschberger ML. Survival analysis: techniques for censored and truncated data. New York, NY: Springer-Verlag, 1997.

38. Efron B. Censored data and the bootstrap. J Am Stat Assoc 1981;76:312-19.

39. U.S. Department of Defense. 2012 MHS stakeholders report. http:// www.health.mil/Reference-Center/Reports/2012/12/31/ 2012-MHS-Stakeholders-Report (accessed 20 Dec 2013). 